\title{
Mirtazapine use may increase the risk of hypothyroxinemia in patients affected by major depressive disorder
}

\author{
Ying Zhao ${ }^{1}$, Na Wang ${ }^{1}$, Shi Wu Wen ${ }^{2}$, Mingcan Li $^{1}$, Yuan Yuan ${ }^{1}$, Ravi Retnakaran ${ }^{3}$, \\ Ruiling Zhang ${ }^{1}$, and Desheng Zhai ${ }^{1}$ \\ ${ }^{1}$ Xinxiang Medical University \\ ${ }^{2}$ University of Ottawa Faculty of Medicine \\ ${ }^{3}$ University of Toronto
}

January 26, 2021

\begin{abstract}
Background Hypothyroxinemia, i.e. Low free T4 with normal TSH level, which overlaps, to a great extent, with the laboratory criteria of central hypothyroidism, could be easily neglected, if attention is paid only to patients with elevated TSH. We aimed to assess the association between mirtazapine use and hypothyroxinemia in patients affected by major depressive disorder. Methods We conducted a retrospective cohort study in the Second Affiliated Hospital of Xinxiang Medical University between January 2016 and December 2018. Patients affected by major depression disorder and admitted to the hospital for treatment during the study period and had thyroid tests at admission and after treatment were included. Patients with abnormal thyroid function at baseline or received mood stabilizers or quetiapine during hospitalization were excluded. Mirtazapine use was the exposure measure, and hypothyroxinemia was as the primary outcome of this study. Log-binomial model was used to estimate the association between mirtazapine use and hypothyroxinemia, after adjusting for potential confounding factors. Results A total of 220 eligible patients were included in the final analysis. Of them, 88 used mirtazapine. The incidence of hypothyroxinemia in patients who used mirtazapine was higher (37.5\%) than those patients who did not use (19.7\%). The relative risk of developing hypothyroxinemia was 1.64 (95\% confidence interval: 1.31-1.78) for mirtazapine use, after adjusting for confounding factors. Conclusion Mirtazapine use was associated with the risk of developing hypothyroxinemia. Clinicians should be aware that hypothyroxinemia may be neglected in patients treated by mirtazapine due to attention paid only to those with elevated TSH.
\end{abstract}

Mirtazapine use may increase the risk of hypothyroxinemia in patients affected by major depressive disorder Running title: Mirtazapine and hypothyroxinemia

Ying Zhao ${ }^{\mathrm{a}, \mathrm{b}, \mathrm{c} \text { \#, Na Wang }}{ }^{\mathrm{b}, \mathrm{c}}$ \#, Shi Wu Wen ${ }^{\mathrm{d}, \mathrm{e}, \mathrm{f}}$, Mingcan Li b,c , Yuan Yuan b,c, Ravi Retnakaran ${ }^{\mathrm{g}, \mathrm{h}}$, Ruiling Zhang ${ }^{\mathrm{a}^{*}}$, Desheng Zhai ${ }^{{ }^{*}}$

\# Ying Zhao and Na Wang should be considered joint first author.

* Correspondence to: R. Zhang, Xinxiang Medical University, The Second Affiliated Hospital, Xinxiang 453002, China. zhangruilingxx@163.com

* Correspondence to: D. Zhai, School of Public Health, Xinxiang Medical University, Xinxiang 453003, China. zds@xxmu.edu.cn

\section{Affiliations}

${ }^{a}$ Xinxiang Medical University, The Second Affiliated Hospital, Xinxiang, 453002, China 
b School of Pharmacy, Xinxiang Medical University, Xinxiang, 453003, China

c Xinxiang Key Laboratory of Clinical psychopharmacology, Xinxiang, 453003, China

d OMNI Research Group, Clinical Epidemiology Program, Ottawa Hospital Research Institute, Ottawa, Canada;

e School of Epidemiology and Public Health, University of Ottawa Faculty of Medicine, Ottawa, Canada;

${ }^{\mathrm{f}}$ Department of Obstetrics, Gynecology, and Newborn Care, University of Ottawa Faculty of Medicine, Ottawa, Canada;

g Division of Endocrinology and Metabolism, University of Toronto, Toronto, Canada;

${ }^{\mathrm{h}}$ Lunenfeld-Tanenbaum Research Institute, Mount Sinai Hospital, Toronto, Canada

i School of Public Health, Xinxiang Medical University, Xinxiang, 453003, China

Principal Investigator statement

The authors confirm that the Principal Investigator for this paper is Ruiling Zhang and that he had direct clinical responsibility for patients.

\section{Author Contributions}

Conceived and designed the study: Desheng Zhai, Shi Wu Wen, Ravi Retnakaran. Participated in data collection and analysis: Ying Zhao, Na Wang, Mingcan Li, Yuan Yuan, Ruiling Zhang. Wrote the first draft of the manuscript: Ying Zhao. All authors contributed to refinement of the study protocol and contributed to, and approved, the final manuscript. Author R. Zhang and D. Zhai have full access to all of the data in the study and take responsibility for the integrity of the data and the accuracy of the data analysis.

\section{Ethics approval statement}

Approval by the Ethics Committee of the Second Affiliated Hospital of Xinxiang Medical University was obtained prior to the commencement of the study.

\section{Funding information}

This work was supported by the Henan Key Science and Technologies Development Program [No.202102310084] (To Desheng Zhai), the Support Project of Scientific and Technological Innovation Team in Universities of Henan Province [No. 20IRTSTHN027] (To Ying Zhao). Partly supported by Henan Key Medical Science and Technologies Development Program [No. SBGJ2018058] (To Ying Zhao).

\section{Conflict of Interest}

None

\section{Data availability statement}

De-identified individual participant data for the study, the study protocol, will be available to investigators for individual participant data meta-analyses that have been approved by independent review committees. Proposals for use of data and requests for access should be directed to zds@xxmu.edu.cn and zhangruilingxx@163.com.

To gain access, researchers will need to sign a data access agreement with the study sponsor (Xinxiang Medical University, The Second Affiliated Hospital, Xinxiang, China).

\section{What is already known about this subject}

It is known that central neurotransmitters system regulates/influences the hypothalamic-pituitary-thyroid axis. Activation of central dopaminergic and serotonergic system has been reported to inhibit hypothalamicpituitary-thyroid axis, reduce TSH releasing and thyroid hormones and may therefore result in hypothyrox- 
inemia (normal TSH concentrations and a disproportionately low concentration of free T4). The laboratory criteria of hypothyroxinemia, defined as "normal TSH and Low free T4", overlaps, to a great extent, with the laboratory criteria of central hypothyroidism (low or low-to-normal TSH concentrations and a disproportionately low concentration of free T4). However, hypothyroxinemia or central hypothyroidism could be easily ignored in patients treated with mirtazapine, a noradrenergic and specific serotonergic antidepressant, if attention is paid to only patients with elevated TSH.

We have conducted a thorough search of literature on PubMed, using a combination of "mirtazapine" AND "thyrotropin OR thyroid-stimulating hormone OR TSH OR thyroxine OR free T4 OR Thyroid function OR hypothyroxinemia OR hypothyroidism". This search found only one relevant paper that assessed thyroid function in 17 outpatients affected by major depressive disorder and treated by mirtazapine. A significant decrease in free T4 but no change in TSH was observed in that study. However, that study did not report whether free T4 was dropped to the cutoff level defining hypothyroxinemia. Most importantly, no comparison with non-mirtazapine users among patients affected by major depressive disorder was made.

\section{What this study adds}

Our study found that mirtazapine use for patients affected by major depressive disorder may increase the risk of hypothyroxinemia.

The finding of increased risk of developing hypothyroxinemia after mirtazapine treatment has significant clinical implication. Clinicians should be aware that thyroid function insufficiency may be ignored in patients treated by mirtazapine due to attention paid only to those with elevated TSH and failure to recognize hypothyroxinemia.

\section{Abstract}

Background Hypothyroxinemia, i.e. Low free T4 with normal TSH level, which overlaps, to a great extent, with the laboratory criteria of central hypothyroidism, could be easily neglected, if attention is paid only to patients with elevated TSH. We aimed to assess the association between mirtazapine use and hypothyroxinemia in patients affected by major depressive disorder.

Methods We conducted a retrospective cohort study in the Second Affiliated Hospital of Xinxiang Medical University between January 2016 and December 2018. Patients affected by major depression disorder and admitted to the hospital for treatment during the study period and had thyroid tests at admission and after treatment were included. Patients with abnormal thyroid function at baseline or received mood stabilizers or quetiapine during hospitalization were excluded. Mirtazapine use was the exposure measure, and hypothyroxinemia was as the primary outcome of this study. Log-binomial model was used to estimate the association between mirtazapine use and hypothyroxinemia, after adjusting for potential confounding factors.

Results A total of 220 eligible patients were included in the final analysis. Of them, 88 used mirtazapine. The incidence of hypothyroxinemia in patients who used mirtazapine was higher $(37.5 \%)$ than those patients who did not use (19.7\%). The relative risk of developing hypothyroxinemia was 1.64 (95\% confidence interval: 1.31-1.78) for mirtazapine use, after adjusting for confounding factors.

Conclusion Mirtazapine use was associated with the risk of developing hypothyroxinemia. Clinicians should be aware that hypothyroxinemia may be neglected in patients treated by mirtazapine due to attention paid only to those with elevated TSH.

Keywords: mirtazapine, thyroid function, hypothyroxinemia, major depressive disorder, acute episode

\section{Introduction}

Thyroid function insufficiency can be classified as primary (due to thyroid hormone deficiency) and central (due to thyroid-stimulating hormone (TSH) deficiency or thyrotropin-releasing hormone (TRH) deficiency)

1. In general, central hypothyroidism is rare, with incidence estimated at $1: 80,000$ to $1: 120,000$ in the 
general population, and accounts for less than $1 / 100$ cases of hypothyroidism ${ }^{1}$. Primary hypothyroidism is defined as TSH concentrations above the reference range and free thyroxine (free T4) concentrations below the reference range ${ }^{1}$. In general, the relationship between serum TSH and free T4 is such that a small decrease in free T4 can result in a relatively large increase in serum TSH, which can subsequently lead to a TSH level that is above the reference range while the free T4 level is still within the reference range ${ }^{2}$. In cases of progression to primary hypothyroidism, TSH level typically continues to increase and free T4 level falls below the reference range ${ }^{1,2}$. Central hypothyroidism is characterized by a defect in thyroid hormone secretion due to insufficient stimulation by $\mathrm{TSH}^{1}$. Biochemically, central hypothyroidism is defined by low-to-normal TSH concentrations and a disproportionately low concentration of free $\mathrm{T} 4{ }^{1}$. The laboratory criteria of hypothyroxinemia, defined as "normal TSH and Low free T4", overlaps, to a great extent, with the laboratory criteria of central hypothyroidism ${ }^{3-5}$.

Circulating thyroid hormones concentrations are regulated by the hypothalamic-pituitary-thyroid axis. TSH controls all aspects of thyroid hormone synthesis and release. Secretion of TSH is stimulated by TRH and inhibited by negative feedback through thyroid hormones ${ }^{6}$. It is known that central neurotransmitters system regulates/influences the hypothalamic-pituitary-thyroid axis ${ }^{3,5,7}$. Activation of the central dopaminergic and serotonergic system has been reported to inhibit hypothalamic-pituitary-thyroid axis, resulting in decrease in the release of TSH and thyroid hormones ${ }^{3,5,7}$.

Mirtazapine is a noradrenergic and specific serotonergic antidepressant. It is particularly effective in alleviating depressed mood and relieving anxiety and sleep-disturbance symptoms, with lower frequencies of drug-drug interaction. As a result, it is often used as an adjunctive antidepressant for moderate to severe major depressive disorder ${ }^{8,9}$. The current clinical literature regarding mirtazapine and thyroid function is scarce, with only one study by Gambi et al in 17 outpatients affected by major depressive disorder ${ }^{10}$. However, Gambi's study did not examine whether patients developed hypothyroxinemia or central hypothyroidism. Most importantly, no comparison with no mirtazapine user was made in the Gambi's study ${ }^{10}$.

Clinical presentation of thyroid function insufficiency varies greatly and generally without symptom specificity $^{1}$. Combined with the fact that symptoms of thyroid function insufficiency and major depressive disorder overlap in many aspects such as fatigue, depression, and weakness ${ }^{11}$, the evaluation of thyroid function in patients treated by antidepressants is predominantly biochemical ${ }^{1}$. However, because central hypothyroidism is rare, attention is typically paid only on patients with elevated TSH, and central hypothyroidism or hypothyroxinemia may be largely neglected ${ }^{3}$. Thyroid function insufficiency is associated with poor response to antidepressant therapies in patients with major depressive disorder ${ }^{12-14}$. Failure to recognize hypothyroxinemia or central hypothyroidism may result in neglected thyroid dysfunction in individuals treated by antidepressants, which may lead to poor response to treatment. Thyroxine has been used as a synergist for treatment-refractory major depression disorder ${ }^{15}$. Lower serum level of free thyroxine is also associated with more affective episodes and greater severity of depression in mood disorders ${ }^{16}$.

We have therefore conducted a retrospective cohort study to assess the association between mirtazapine use and hypothyroxinemia in patients affected by major depressive disorder. Because the evaluation of thyroid function was based on the reference ranges of TSH and thyroxine, we adopted the term "hypothyroxinemia" for the "normal TSH level and a disproportionately low level of free T4" in this study.

\section{Methods}

\section{Study design and study population}

This is a retrospective cohort study conducted between January 2016 and December 2018 in the Second Affiliated Hospital of Xinxiang Medical University (also known as Henan province Mental Health Center). Approval by the Ethics Committee of the Second Affiliated Hospital of Xinxiang Medical University was obtained prior to the commencement of the study.

Patients affected by major depression disorder and admitted to the participating hospital for antidepressant treatment during the study period with thyroid function tests made at admission and during hospitalization 
were included into this study. The diagnosis of major depressive disorder was made by two staff psychiatrists and confirmed by a third senior psychiatrist, following the "National Guidelines of Diagnosis and Treatment for Mental Disorders" in China and was coded by International Classification of Diseases, $10^{\text {th }}$ revision (ICD10).

Patients with a thyroid function test outside of the normal range (i.e., TSH: $0.27-4.20 \mathrm{mU} / \mathrm{L}$ and free T4: $12.00-22.00 \mathrm{pmol} / \mathrm{L}$ ) or on l-thyroxine treatment at admission were excluded. Patients who met the following criteria were further excluded: 1) ongoing pregnancy or lactation, 2) alterations of hepatic or renal function, or other serious medical condition, 3) history of alcohol or other substance dependence, 4) primary thyroid diseases, or known medical conditions that affect thyroid function, or 5) used mood stabilizers (i.e. lithium, valproic acid, and oxcarbazepine et al.) or quetiapine during hospitalization.

\section{Outcome measures}

Hypothyroxinemia, defined as $0.27[?] \mathrm{TSH}[?] 4.2 \mathrm{mU} / \mathrm{L}$ and $\mathrm{FT} 4<12.00 \mathrm{pmol} / \mathrm{L}$, was the primary outcome of this study. Secondary outcomes included in this study were: The levels of TSH and thyroid hormones, subclinical hypothyroidism (TSH $>4.2 \mathrm{mU} / \mathrm{L}$ and 12.00 [?]free T4[?]22.00 pmol/L), and overt hypothyroidism $(\mathrm{TSH}>4.2 \mathrm{mU} / \mathrm{L}$ and free $\mathrm{T} 4<12.00 \mathrm{pmol} / \mathrm{L})$.

Exposure measure

Mirtazapine use during hospitalization for treatment for major depression disorder was the exposure measure of this study, with any mirtazapine use as the exposed group and no mirtazapine use as the non-exposed group.

\section{Laboratory tests}

The laboratory reference ranges of thyroid hormones were: TSH: $0.27-4.20 \mathrm{mU} / \mathrm{L}, \mathrm{T} 4: 66.00-181.00 \mathrm{nmol} / \mathrm{L}$, free T4: $12.00-22.00 \mathrm{pmol} / \mathrm{L}$, T3: $1.30-3.10 \mathrm{nmol} / \mathrm{L}$, FT3: 3.10-6.80 pmol/L. Standardized criteria of thyroid function were based on the laboratory reference ranges, as follows: normal thyroid function: $0.27[?] \mathrm{TSH}[?] 4.2$ $\mathrm{mU} / \mathrm{L}$ and 12.00[?]free T4[?]22.00 pmol/L. Serum samples were collected between 6:00 and 8:00 AM following an overnight fasting. The tests were completed on the same day at the hospital laboratory. Chemiluminescence procedures (Cobas E610 or 411, Roche, Basel, Switzerland) were employed to determine the thyroid hormones by using Elecsys (TSH, T4, free T4, T3, FT3) kits (Roche Diagnostics Shanghai Ltd, China).

\section{Demographic and clinical data collection}

Demographic and clinical data including diagnosis, illness duration, and treatment for major depression disorder, and value and time at measurement of thyroid functions were collected through review of patients' medical charts.

\section{Statistical Analysis}

Statistical analysis was conducted using the Statistical Package for Social Sciences (IBM SPSS, version 25.0). We first compared the distribution of baseline characteristics and we then compared outcomes between the exposed and the non-exposed groups. Means and standard deviations (SDs) or median and interquartile ranges (IQRs) were used for continuously distributed variables, and t-test or median Mann-Whitney U test were used for statistical significance as appropriate. Percentage was used for categorical variables and $\chi^{2}$ test was used for statistical significance.

Adjusted relative risk (RR) and 95\% CI were estimated by log-binomial model to assess the independent association of mirtazapine use with risk of developing hypothyroxinemia (with no mirtazapine use as the reference). Multiple linear regression model was used to assess the association of mirtazapine use with change in free $\mathrm{T} 4$ level $\left[\Delta\right.$ free $\mathrm{T} 4_{(2-1)}$ : free $\mathrm{T} 4$ value after exposure ${ }_{(2)}-$ free $\mathrm{T} 4$ value at baseline (1) $]$. Potential confounding variables included in the models were age, gender, prior drug exposure (no prior use of psychiatric medications eg. antipsychotics, mood stabilizers and antidepressants for a minimum 4 months 
before admission, were defined as drug free. No drug free versus drug free), baseline free T4 level, duration of treatment, the use of other kinds of antidepressants, and concomitant non-antidepressant medications.

\section{Results}

A total of 220 patients met the eligibility criteria and were included in the final analysis (Figure 1). Among them, $88(40 \%)$ used mirtazapine as an adjunctive antidepressant and $132(60 \%)$ did not use. The majority of patients $(71.8 \%)$ were female. The mean age of the patients was 47.5 (SD:15.23) years and median duration of illness was 12 months (IQR: 56). The mean interval between the two thyroid function tests (baseline and after treatment) was 35.6 days (SD 22.54).

\section{Comparison of baseline characteristics between the two study groups}

Table 1 compares the distribution of demographics and clinical characteristics of two groups. There were no significant differences in age, gender, duration of illness, and uses of antipsychotics, benzodiazepines, anxiolytics, and selective serotonin reuptake inhibitors (SSRIs) between the two groups. The uses of serotoninnorepinephrine reuptake inhibitors (SNRIs) or trazodone were higher in the non-mirtazapine group than those in the mirtazapine group $(P<0.01)$. There were no differences between the two groups with respect to baseline level of thyroid function measurements (Table 1). On the other hand, baseline free T4 levels were negatively associated with risk of hypothyroxinemia, with adjusted $\mathrm{RR}=0.88$ (95\% CI: 0.86-0.90) for $1 \mathrm{pmol} / \mathrm{L}$ increment in baseline free $\mathrm{T} 4$.

\section{Analysis of association between mirtazapine use and occurrence of hypothyroxinemia}

The rate of hypothyroxinemia in the mirtazapine group $(33 / 88,37.5 \%)$ was higher than in the nonmirtazapine group $(26 / 132,19.7 \%)$. After adjusting for age, gender, duration of treatment, baseline free T4 level, use of other antidepressants (SSRIs, SNRIs or trazodone), use of non-antidepressant medications and other potential confounders, patients who received mirtazapine as an adjunctive antidepressant were more likely to develop hypothyroxinemia compared with those who did not receive mirtazapine (adjusted RR: 1.64, 95\% CI: 1.31-1.78) (Table 2).

\section{Analysis of association between mirtazapine use and other thyroid dysfunctions}

In the mirtazapine group, the reduction in $\mathrm{T} 4$ was $15.63 \mathrm{nmol} / \mathrm{L}$, and was $3.22 \mathrm{pmol} / \mathrm{L}$ in free T4, which were significantly greater than those in non-mirtazapine group $(t=-3.053, P=0.003$, and $t=-2.861, P=0.005$, for $\Delta \mathrm{T} 4$ and $\Delta$ free $\mathrm{T} 4$, respectively) (Table 3 ). In the multiple linear regression model, mirtazapine use was statistically significantly associated with greater reduction in free T4 level $\left[\left(\Delta\right.\right.$ free $\left.\left.\mathrm{T} 4_{(2-1)}\right)\right]$ after adjusting for confounders, with adjusted B -1.14 (95\% CI: $\left.-1.88^{\sim}-0.41, P=0.002\right)$. The occurrence of subclinical and overt hypothyroidism was similar in the two groups (Table 3 ).

\section{Discussion}

In this retrospective cohort study, we demonstrated that the risk of developing hypothyroxinemia in patients who used mirtazapine was $64 \%$ higher than patients who did not use, after adjusting for important confounders.

There are several strengths in this study. To the best of our knowledge, this is the first study to report an association between mirtazapine and increased risk of hypothyroxinemia. The large sample size of our study helped to generate stable estimates. The use of electronic inpatients records provided complete measurements of thyroid function: T4, free T4, and TSH at baseline and during treatment. As a result, we could have complete ascertainment of hypothyroxinemia. Furthermore, we excluded patients who had thyroid dysfunction at baseline or who received mood stabilizers (i.e. lithium, valproic acid, and oxcarbazepine) or quetiapine during hospitalization and adjusted for a number of important confounding factors including the baseline level of free T4 and TSH and the use of other kinds of antidepressants during the treatment. Thus, the impact of prior thyroid dysfunction or other psychiatric medications on the observed association between mirtazapine use and hypothyroxinemia would have been minimized. 
Limitations of this study should be acknowledged. First, we had no dynamic monitoring information of thyroid function. Although we have collected data on serum thyroid hormone levels at baseline and during hospitalization for antidepressants treatment, no additional follow up tests were performed. As a result, we could not determine whether the effect of mirtazapine on thyroid function persists after discharge from hospital.

Second, the clinical symptoms/signs of thyroid dysfunction were not available in this study. The laboratory criteria of hypothyroxinemia, defined as "normal TSH and Low free T4", overlaps, to a great extent, with the laboratory criteria of central hypothyroidism ${ }^{3-5}$. Based on available data, we could not definitely differentiate hypothyroxinemia from central hypothyroidism. We adopted the term "hypothyroxinemia" for the "lower free T4 (¡12.00 pmol/L) and normal TSH level” in our study.

Case reports ${ }^{17-21}$ and clinical studies ${ }^{22-26}$ suggested that antidepressants other than mirtazapine may also be associated with thyroid abnormalities. However, no conclusion could be drawn from these studies due to small sample size ${ }^{23-26}$, cross-sectional study design ${ }^{22}$, incomplete laboratory tests ${ }^{20,21,26}$. and lack information whether hormone concentrations reached cut off values ${ }^{23,24,26}$. On the other hand, our study found that the incidence of hypothyroxinemia in patients treated by SSRIs, SNRIs, or combined treatment in nonmirtazapine group was similar (data available upon request). Our findings, in principle, were consistent with previous studies/reviews that SSRIs and SNRIs reduced T4/FT4 levels but did not significantly affect TSH level ${ }^{27}$.

Compared with other treatments for major depressive disorder, the impact of mirtazapine treatment on thyroid function has not been well investigated. To date, only one study reported a significant decrease in free T4 in 17 outpatients affected by major depressive disorder after mirtazapine treatment for 6 months ${ }^{10}$. No change in TSH was observed and no comparison with non-mirtazapine users was made in that study ${ }^{10}$. Although that study did not report if free T4 was dropped to the cutoff level defining hypothyroxinemia, the significant decrease in free T4 after mirtazapine treatment ${ }^{10}$ provides some support to our finding on mirtazapine and hypothyroxinemia association. That previous study also reported a significant increase in FT3 after mirtazapine treatment ${ }^{10}$, which was not observed in our study. On the other hand, our study observed that the degree of reduction in $\mathrm{T} 4$ and free $\mathrm{T} 4$ after mirtazapine treatment was much higher than the reductions in T3 and FT3, which suggests that peripheral conversion of T4 to T3 could be affected by mirtazapine treatment, and this process may occur mostly in liver, heart, muscle and gut, rather than in thyroid $^{6}$. Increased peripheral conversion of T4 to T3 may also be a consequence of a compensatory response to lower function of hypothalamic-pituitary-thyroid axis, due to changes in thyroid hormone metabolism that serve to maintain normal circulating levels of T3 until the late stages of dysfunction ${ }^{6}$.

More than $99 \%$ of circulating thyroid hormones is bound to serum proteins. It is reported that commercially available testing kits might show inaccurately low free thyroxine concentrations in patients treated with drugs which have the capacity to displace thyroid hormone from binding proteins, and it is suggested that serum dilution in assays could reduce free thyroxine readout ${ }^{6}$. However, we observed that the degree of reduction was very similar in both T4 and free T4. For example, the reduction was $18.77 \%$ in T4 and $21.09 \%$ in free T4 in the mirtazapine group. It is known that serum dilution in assays would not affect T4 readout ${ }^{6}$. Therefore, in this case, the low serum free T4 levels observed in patients treated with mirtazapine may not be due to artifactual laboratory effects.

The key to maintain the level of serum free thyroxine is the negative feedback regulation, an appropriate TSH synthesis and release in response to serum thyroid hormone level changes ${ }^{6}$. Activation of the central neurotransmitters system, such as dopaminergic and serotonergic system, has been reported to inhibit hypothalamic-pituitary-thyroid axis ${ }^{3,5,7}$. The increased risk of hypothyroxinemia after mirtazapine treatment could be explained by the central effect of mirtazapine on hypothalamic/pituitary prevents the compensatory increase in TSH in response to low T4, thereby ultimately presenting as higher incidence of hypothyroxinemia in patients treated by mirtazapine. It has been reported that antidepressants (other than mirtazapine), which improve central dopaminergic and serotonergic system, seem to decrease T4/free T4 levels but maintaining TSH level ${ }^{27}$. Our study found that after adjusting for other antidepressant treatments, 
the association between mirtazapine treatment and hypothyroxinemia remained significant.

The finding of increased risk of developing hypothyroxinemia after mirtazapine treatment may have significant clinical implication. ${ }^{13}$ Symptoms of thyroid dysfunction and major depressive disorder overlap in many aspects such as depressive mood, fatigue and weakness ${ }^{11}$. Thus, hypothyroxinemia could be easily ignored in major depressive disorder treated with mirtazapine, if attention is paid only to those with elevated TSH.

In addition, clinical studies indicate that thyroid hormone supplementation can improve the response rate and can also convert patients with refractory depression to responders ${ }^{15}$. The beneficial effects of thyroid hormone supplementation has suggested that a central hypothyroidism may exist in such patients ${ }^{15}$. Failure to recognize hypothyroxinemia or central hypothyroidism may result in ignored thyroid function insufficiency, which may lead to poor response to treatment in individuals with major depression disorder. It may therefore be important to identify thyroid function insufficiency during antidepressants treatment for major depressive disorder, especially in patients receiving mirtazapine as an adjunctive antidepressant.

\section{Conclusion}

Our study based on a large cohort of patients affected by major depressive disorder suggests that mirtazapine treatment may be associated with the increased risk of developing hypothyroxinemia. Further investigations are needed to clarify the clinical relevance of this association and its long-term impact on the treated patients.

\section{Figure legend}

\section{Figure 1 Data source and flow chart.}

\section{References}

1. Chaker L, Bianco AC, Jonklaas J, Peeters RP. Hypothyroidism. Lancet (London, England) $2017 ; 390$ (10101): 1550-62.

2. Peeters RP. Subclinical Hypothyroidism. The New England journal of medicine 2017;376 (26): 2556-65.

3. Beck-Peccoz P, Rodari G, Giavoli C, Lania A. Central hypothyroidism - a neglected thyroid disorder.Nature reviews Endocrinology 2017; 13 (10): 588-98.

4. Lania A, Persani L, Beck-Peccoz P. Central hypothyroidism. Pituitary 2008; 11 (2): 181-6.

5. Slag MF, Morley JE, Elson MK, Crowson TW, Nuttall FQ, Shafer RB. Hypothyroxinemia in critically ill patients as a predictor of high mortality. Jama 1981;245 (1): 43-5.

6. Burch HB. Drug Effects on the Thyroid. The New England journal of medicine 2019;381 (8): 749-61.

7. Haugen BR. Drugs that suppress TSH or cause central hypothyroidism. Best practice $E$ research Clinical endocrinology 85 metabolism 2009; 23 (6): 793-800.

8. Kent JM. SNaRIs, NaSSAs, and NaRIs: new agents for the treatment of depression. Lancet (London, England) 2000; 355 (9207): 911-8.

9. Graves SM, Rafeyan R, Watts J, Napier TC. Mirtazapine, and mirtazapine-like compounds as possible pharmacotherapy for substance abuse disorders: evidence from the bench and the bedside. Pharmacology $\mathcal{E}^{\circ}$ therapeutics 2012;136 (3): 343-53.

10. Gambi F, De Berardis D, Sepede G, et al. Effect of mirtazapine on thyroid hormones in adult patients with major depression. International journal of immunopathology and pharmacology 2005; 18 (4): 737-44.

11. Dickerman AL, Barnhill JW. Abnormal thyroid function tests in psychiatric patients: a red herring? The American journal of psychiatry 2012; 169 (2): 127-33.

12. Cole DP, Thase ME, Mallinger AG, et al. Slower treatment response in bipolar depression predicted by lower pretreatment thyroid function. The American journal of psychiatry 2002; 159 (1): $116-21$. 
13. Abulseoud O, Sane N, Cozzolino A, et al. Free T4 index and clinical outcome in patients with depression. Journal of affective disorders 2007; 100 (1-3): 271-7.

14. Pae CU, Mandelli L, Han C, et al. Thyroid hormones affect recovery from depression during antidepressant treatment. Psychiatry and clinical neurosciences 2009;63 (3): 305-13.

15. Aronson R, Offman HJ, Joffe RT, Naylor CD. Triiodothyronine augmentation in the treatment of refractory depression. A meta-analysis. Archives of general psychiatry 1996;53 (9): 842-8.

16. Bauer MS, Whybrow PC, Winokur A. Rapid cycling bipolar affective disorder. I. Association with grade I hypothyroidism. Archives of general psychiatry 1990;47 (5): 427-32.

17. Harel Z, Biro FM, Tedford WL. Effects of long term treatment with sertraline (Zoloft) simulating hypothyroidism in an adolescent. The Journal of adolescent health : official publication of the Society for Adolescent Medicine 1995;16 (3): 232-4.

18. Eker SS, Akkaya C, Ersoy C, Sarandol A, Kirli S. Reversible escitalopram-induced hypothyroidism. General hospital psychiatry 2010; 32 (5): 559.e5-7.

19. Lai J, Xu D, Peterson BS, et al. Reversible Fluoxetine-Induced Hyperthyroidism: A Case Report.Clinical neuropharmacology 2016; 39 (1): 60-1.

20. Takahashi M, Sawayama E, Sawayama T, Miyaoka H. Reversible paroxetine-induced symptomatic hypothyroidism. Pharmacopsychiatry 2007; 40 (5): 201-2.

21. Mazokopakis EE, Karefilakis CM, Starakis IK. Escitalopram-induced subclinical hypothyroidism. A case report. Hormones (Athens, Greece) 2012; 11 (1): 101-3.

22. Fugger G, Dold M, Bartova L, et al. Comorbid thyroid disease in patients with major depressive disorder - results from the European Group for the Study of Resistant Depression (GSRD). European neuropsychopharmacology : the journal of the European College of Neuropsychopharmacology 2018; 28 (6): $752-60$.

23. de Carvalho GA, Bahls SC, Boeving A, Graf H. Effects of selective serotonin reuptake inhibitors on thyroid function in depressed patients with primary hypothyroidism or normal thyroid function. Thyroid : official journal of the American Thyroid Association 2009; 19 (7): 691-7.

24. Eker SS, Akkaya C, Sarandol A, Cangur S, Sarandol E, Kirli S. Effects of various antidepressants on serum thyroid hormone levels in patients with major depressive disorder.Progress in neuro-psychopharmacology $\mathcal{E}$ biological psychiatry2008; 32 (4): 955-61.

25. Shelton RC, Winn S, Ekhatore N, Loosen PT. The effects of antidepressants on the thyroid axis in depression. Biological psychiatry 1993; 33 (2): 120-6.

26. König F, Hauger B, von Hippel C, Wolfersdorf M, Kaschka WP. Effect of paroxetine on thyroid hormone levels in severely depressed patients. Neuropsychobiology 2000;42 (3): 135-8.

27. Bou Khalil R, Richa S. Thyroid adverse effects of psychotropic drugs: a review. Clinical neuropharmacology 2011; 34 (6): 248-55.

\section{Hosted file}

Figure1 Data source and flow chart.pdf available at https://authorea.com/users/391933/ articles/505937-mirtazapine-use-may-increase-the-risk-of-hypothyroxinemia-in-patientsaffected-by-major-depressive-disorder

\section{Hosted file}

Tables.pdf available at https://authorea.com/users/391933/articles/505937-mirtazapine-usemay-increase-the-risk-of-hypothyroxinemia-in-patients-affected-by-major-depressive- 
disorder 